\title{
The value of GRASP on DCE-MRI for assessing response to neoadjuvant chemotherapy in patients with esophageal cancer
}

Yanan Lư ${ }^{1 \dagger}$, Ling Ma ${ }^{2 \dagger}$, Jianjun Qin ${ }^{3,4 \dagger}$, Zhaoqi Wang ${ }^{1}$, Jia Guo ${ }^{1}$, Yan Zhao ${ }^{1}$, Hongkai Zhang ${ }^{1}$, Xu Yan ${ }^{5}$, Hui Liu', Hailiang Li', Ihab R. Kamel ${ }^{6}$ and Jinrong $\mathrm{Qu}^{1^{*}}$ (D)

\begin{abstract}
Background: To compare the value of two dynamic contrast-enhanced Magnetic Resonance Images (DCE-MRI) reconstruction approaches, namely golden-angle radial sparse parallel (GRASP) and view-sharing with golden-angle radial profile (VS-GR) reconstruction, and evaluate their values in assessing response to neoadjuvant chemotherapy $(\mathrm{nCT})$ in patients with esophageal cancer (EC).
\end{abstract}

Methods: EC patients receiving $\mathrm{nCT}$ before surgery were enrolled prospectively. DCE-MRI scanning was performed after nCT and within 1 week before surgery. Tumor Regression Grade (TRG) was used for chemotherapy response evaluation, and patients were stratified into a responsive group $($ TRG $1+2)$ and a non-responsive group $($ TRG $3+4+$ 5). Wilcoxon test was utilized for comparing GRASP and VS-GR reconstruction, Kruskal-Wallis and Mann-Whitney test was performed for each parameter to assess response, and Spearman test was performed for analyzing correlation between parameters and TRGs, as well as responder and non-responder. The receiver operating characteristic (ROC) was utilized for each significant parameter to assess its accuracy between responders and non-responders.

Results: Among the 64 patients included in this cohort (52 male, 12 female; average age of $59.1 \pm 7.9$ years), 4 patients showed TRG1, 4 patients were TRG2, 7 patients were TRG3, 11 patients were TRG4, and 38 patients were TRG5. They were stratified into 8 responders and 56 non-responders.

A total of 15 parameters were calculated from each tumor. With VS-GR, 10/15 parameters significantly correlated with TRG and response groups. Of these, only AUCmax showed moderate correlation with TRG, 7 showed low correlation and 2 showed negligible correlation with TRG. 8 showed low correlation and 2 showed negligible correlation with response groups. With GRASP, 13/15 parameters significantly correlated with TRG and response groups. Of these, 10 showed low correlation and 3 showed negligible correlation with TRG. 11 showed low correlation and 2 showed negligible correlation with TRG. Seven parameters $\left(A \cup C^{*}>0.70, P<0.05\right)$ showed good performance in response groups.

(Continued on next page)

\footnotetext{
* Correspondence: qjryq@126.com

†Yanan Lu, Ling Ma and Jianjun Qin contributed equally to this work.

'Department of Radiology, the Affiliated Cancer Hospital of Zhengzhou University \& Henan Cancer Hospital, 127 Dongming Road, Zhengzhou 450008, Henan, China

Full list of author information is available at the end of the article
}

(c) The Author(s). 2019 Open Access This article is distributed under the terms of the Creative Commons Attribution 4.0 International License (http://creativecommons.org/licenses/by/4.0/), which permits unrestricted use, distribution, and reproduction in any medium, provided you give appropriate credit to the original author(s) and the source, provide a link to the Creative Commons license, and indicate if changes were made. The Creative Commons Public Domain Dedication waiver (http://creativecommons.org/publicdomain/zero/1.0/) applies to the data made available in this article, unless otherwise stated. 
(Continued from previous page)

Conclusions: In patients with esophageal cancer on neoadjuvant chemotherapy, several parameters can

differentiate responders from non-responders, using both GRASP and VS-GR techniques. GRASP may be able to

better differentiate these two groups compared to VS-GR.

Trial registration for this prospective study: ChiCTR, ChiCTR-DOD-14005308. Registered 2 October 2014.

Keywords: Magnetic resonance imaging, Esophageal Cancer, Treatment outcome, Chemotherapy, Neoadjuvant therapy,

\section{Background}

Esophageal cancer (EC) has become the eighth most common cancer, and the incidence rate is rising rapidly worldwide [1]. Squamous cell carcinoma (SCC) is the main pathological type of EC in China, and is a highgrade malignancy with rapid progression, poor response and high recurrence rate $[2,3]$. Moreover, SCC is associated with limited quality of life after surgery, poor prognosis [4] and a high incidence of postoperative morbidity and mortality [5-7]. Ando et al. reported that $\mathrm{nCT}$ before resection is still the main treatment for stages II and III SCC [7, 8]. If local tumor is controlled, $\mathrm{nCT}$ followed by surgical procedures is an optimum treatment strategy, which can improve overall survival for patients with SCC [8]. Predicting response to $\mathrm{nCT}$ accurately helps clinicians to provide the best treatment approach such as modification of $\mathrm{nCT}$, or termination of $\mathrm{nCT}$ to initiate surgical resection $[1,9]$.

18 F-fluorodeoxyglucose positron emission tomography (18 F-FDG-PET) shows to be a promising technique for predicting therapeutic response, but standardizing protocols and the time of scanning is required [10]. Dynamic contrast-enhanced Magnetic Resonance Images (DCEMRI) have the ability to predict an early response in EC following 3 weeks of concurrent chemoradiotherapy in limited cases $[11,12]$. However, it is still challenging to non-invasively predict response to nCT. Recently, goldenangle radial sparse parallel (GRASP) MRI has gained interest, and has been applied to imaging of the liver, rectal cancer and renal cell carcinoma [13-16]. GRASP is capable of reconstructing the acquired data at very high temporal resolution using only a small number of radial spokes for every temporal frame. This enables highresolution free-breathing perfusion imaging with higher in-plane spatial resolution and thinner partitions. This results in near-isotropic resolution, compared with the current view-sharing with golden-angle radial profile (VSGR) reconstruction, without the current imaging constraints of breath-holding techniques [13].

The aim of this study was to compare DCE-MRI with GRASP reconstruction to DCE-MRI with VS-GR reconstruction in assessing response to $\mathrm{nCT}$ in patients with EC and to identify DCE-MRI parameters that can differentiate responders from non-responders.

\section{Methods}

This prospective study was approved by the Ethics Committee of Henan Cancer Hospital (No.20140303), and written informed consent was obtained from all participants. Those patients who received $\mathrm{nCT}$ followed by surgical resection were enrolled. DCE-MRI was performed within 1 week before surgery. All studies were performed between September 2015 and March 2017. The inclusion criteria were following [17]: 1) Patients were confirmed with stage II-III EC by esophagoscopy pathologically $[18,19]$, 2) 2 cycles of nCT before surgery were performed, 3) Imaging and clinical response evaluation were performed at 2 weeks after completing all the treatment. (Fig. 1).

\section{DCE-MRI scanning methods}

DCE-MRI examination was performed on a $3 \mathrm{~T}$ MR scanner (MAGNETOM Skyra, Siemens Healthcare) with dynamic contrast-enhanced Radial VIBE free breathing, and an 18-element body matrix coil and an inbuilt 32element spine matrix coil were used. Radial VIBE sequence parameters were following: TR: $3.98 \mathrm{~ms}$ TE: 1.91 ms, flip angle: $12^{\circ}$, acquisition matrix: $300 \times 300$, FOV: $300 \mathrm{~mm} \times 300 \mathrm{~mm} \times 146 \mathrm{~mm}$, slice thickness: $3 \mathrm{~mm}$, reconstructed image voxel size: $1.0 \times 1.0 \times 3.0 \mathrm{~mm}^{3}$, radial views: 1659 , scanning time: $309 \mathrm{~s}$. A total of 68 period images were collected, and each period included $72 \mathrm{im}$ ages. $10-15 \mathrm{~mL}$ Gadopentetate Dimeglumine Injection $(0.2 \mathrm{ml} / \mathrm{kg}$ of body weight, Omniscan, GE Healthcare) was injected at a rate of $2.5 \mathrm{~mL} / \mathrm{s}$, followed by equal volume of normal saline solution to flush the tube at $20 \mathrm{~s}$ after the beginning of scanning by a MR-compatible automated high-pressure injector (Spectris Solaris EP, Medrad) [17].

\section{Histopathology response}

Pathologic response was assessed as 5 grades according to Tumor Regression Grade (TRG) [20]: TRG 1 (complete regression) showed absence of residual cancer and fibrosis extending through the different layers of the esophageal wall; TRG 2 was characterized by the presence of rare residual cancer cells scattered through bands of fibrosis; TRG 3 was characterized by an increase in the number of residual cancer cells, but fibrosis 


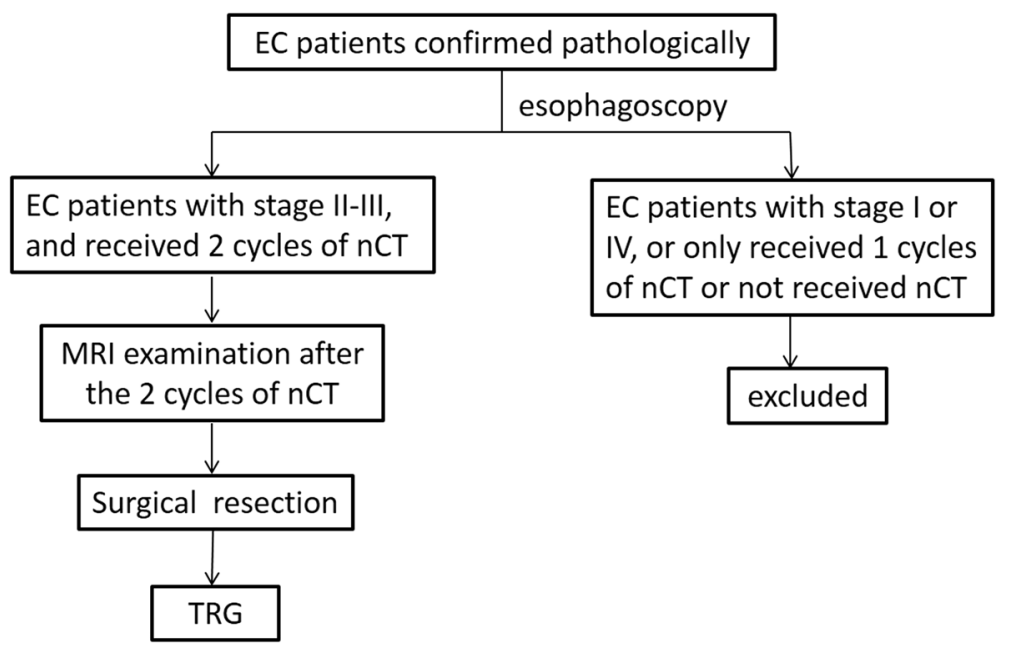

Fig. 1 Flow chart illustrates patient selection process for study cohort

still predominated; TRG 4 showed residual cancer outgrowing fibrosis; and TRG 5 was characterized by absence of regressive changes. They were stratified into a responsive group $(\mathrm{TRG} 1+2)$ and a non-responsive group (TRG3 + 4+5).

\section{Image processing and data analysis}

The radial views (1659 of stack-of-stars views acquired from DCE-MRI) were input into online reconstruction pipeline of view sharing reconstruction and regrouped into 2 sub-frames (sub-frame-1: T0-T61 with a temporal resolution of $2.4 \mathrm{~s}$, sub-frame 2 from T62-T68 with temporal resolution of $21.7 \mathrm{~s}$ ). A home setup of GRASP reconstruction processing pipeline (https:// mrirecon.github.io/bart/) post processed on a Yarra server (https://yarra.rocks) were used for GRASPs offline, with the same data but using a temporal resolution of $4.5 \mathrm{~s}$ (Table 1 ).

The images reconstructed by two different approaches, namely GRASP and VS-GR, were processed by OmniKinetics software (GE Medical, China) to segment the tumor and generate pharmacokinetic parameters respectively. The thoracic aorta was selected to obtain the arterial input function (AIF), since the esophageal artery is not easy to identify. Figure 2 shows the AIFs derived from GRASP and VS-GR reconstructions from the same contrast-enhanced study.

Two radiologists with more than 10 years experiences in thorax radiology segmented the 3D- regions of interest (ROI) manually. The radiologists were blinded to clinical data, and were asked to include the entire tumor on each slice post-nCT, except areas of necrotic degeneration or cystic and normal blood vessels. The pharmacokinetic parameters were generated by using Tofts model.

\section{Statistical analysis}

SPSS Statistics version 22 (IBM Corp., Armonk, NY, USA) were used to perform statistical analysis in this study. Interobserver reproducibility of pharmacokinetic parameters was assessed by inter-class correlation coefficients (ICCs). An ICC > 0.75 was considered good agreement. The Wilcoxon test of was used to compare the various parameters between VS-GR and GRASP

Table 1 Details of reconstruction setting for radial VIBE with golden angle stack-of-stars sampling scheme

\begin{tabular}{lll}
\hline & View-Sharing & GRASP \\
\hline number of acquired views & 1659 & \\
FOV & $300 \mathrm{~mm} \times 300 \mathrm{~mm} \times 146 \mathrm{~mm}$ & \\
spatial resolution & $1.0 \times 1.0 \times 3.0 \mathrm{~mm}^{3}$ & $4.5 \mathrm{~s}$ \\
temporal resolution & $2.4 \mathrm{~s} / 21.7 \mathrm{~s}$ & 68 \\
number of dynamic volumes & 68 & Offline \\
Reconstruction mode & Online & 62 minutes on a CPU server \\
Reconstruction time & N/A & .
\end{tabular}

Note: The temporal resolution of VS-GR means the starting time interval between two phases, however, $90 \%$ of the prior phase was overlapped with this phase. So, although the temporal resolution of VS-GR seems very short, actually it is longer 


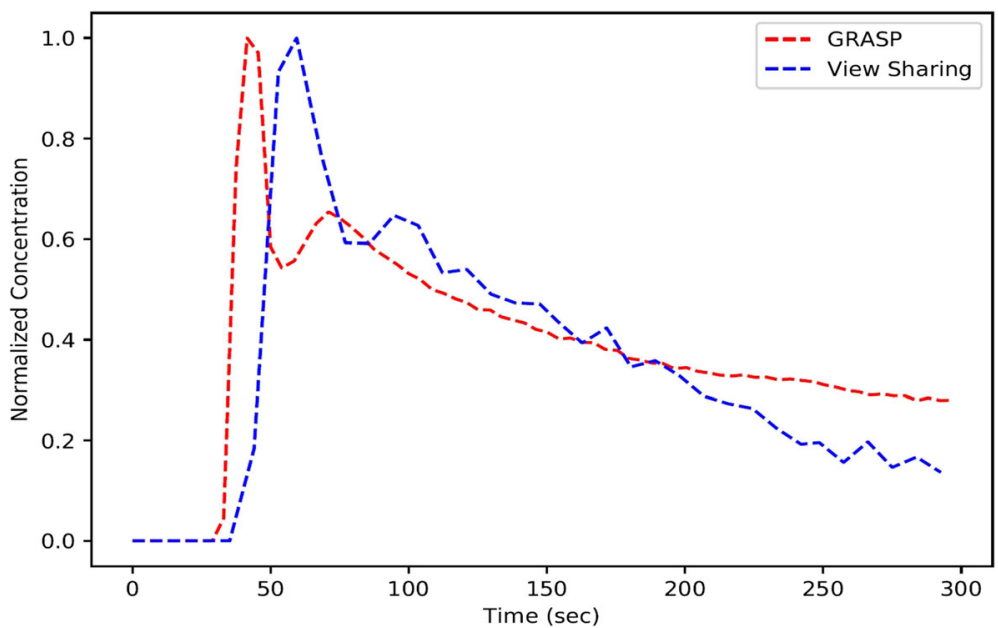

Fig. 2 Arterial contrast concentration curve from GRASP (red) and view-sharing (blue) reconstruction using the same dynamic acquisition. GRASP'S AIF is closer to the true AIF with steeper slope and sharp peak than view-sharing

reconstruction, and Kruskal-Wallis test for DCE-MRI parameters with VS-GR or GRASP reconstruction among the TRG1-5 groups $(P<0.05)$. Mann-Whitney test was for analyzing the differences between responder and non-responder groups. Spearman test was performed for correlation analysis between DCEMRI parameters and TRGs, or response groups. Spearman's correlation coefficients were assessed as follows: a correlation coefficient of $0.90-1.00$ is considered very high; $0.70-0.89$, high; $0.50-0.69$, moderate; $0.30-0.49$, low; and $0-0.29$, negligible [21]. The receiver operating characteristic (ROC) was adopted to assess the value of each parameter in predicting response (AUC" $>0.50, P<0.05)$.

\section{Results}

Among the total of 64 patients $(52$ male, 12 female, average age of $59.1 \pm 7.9$ years), 59 patients had SCC, 2 patients had adenocarcinoma and 3 patients had adenosquamous carcinoma. According to pathologic response, 4 patients showed TRG1, 4 patients were TRG2, 7 patients were TRG3, 11 patients were TRG4, and 38 patients were TRG5. They were stratified into 8 responders and 56 non-responders (Table 2).

ICCs showed the excellence of 15 pharmacokinetic parameters from the two reconstructions as assessed by the two radiologists, and the kappa value was 0.918 .

\section{Comparison of DCE-MRI parameters with VS-GR and GRASP reconstruction groups}

GRASP showed a better AIF curve with steeper slope and sharper peak compared to VS-GR (Fig. 2). A total of 15 pharmacokinetic parameters were extracted from each tumor. 14 of these showed statistically significant difference for both VS-GR and GRASP reconstruction
Table 2 Patients' demographic information and TRG

\begin{tabular}{|c|c|}
\hline Study population & \\
\hline \multicolumn{2}{|l|}{ Gender } \\
\hline Male & 52 \\
\hline Female & 12 \\
\hline Age, years & $59.1 \pm 7.9$ \\
\hline \multicolumn{2}{|l|}{ Clinical T-stage } \\
\hline $\mathrm{T} 1$ & 2 \\
\hline $\mathrm{T} 2$ & 15 \\
\hline T3 & 42 \\
\hline $\mathrm{T} 4$ & 5 \\
\hline \multicolumn{2}{|l|}{ Clinical N-stage } \\
\hline No & 32 \\
\hline N1 & 15 \\
\hline N2 & 15 \\
\hline N3 & 2 \\
\hline \multicolumn{2}{|l|}{ Type } \\
\hline SCC & 59 \\
\hline$A C$ & 2 \\
\hline ASC & 3 \\
\hline \multicolumn{2}{|c|}{ Tumor Regression Grade } \\
\hline 1 & 4 \\
\hline 2 & 4 \\
\hline 3 & 7 \\
\hline 4 & 11 \\
\hline 5 & 38 \\
\hline
\end{tabular}


across the TRG groups. Only plasma volume fraction (Vp) max did not show a significant difference $(P=$ $0.628)$.

\section{Comparison among TRG1-5 for DCE-MRI parameters with VS-GR and GRASP reconstruction}

14/15 DCE-MRI parameters both with VS-GR and with GRASP reconstruction showed significant inter-groups difference by TRG $1-5(P<0.05)$, except for Ve max which showed not significant inter-groups difference by TRG 1-5 (Table 3).

\section{Comparison between responder and non-responder groups for DCE-MRI parameters with VS-GR/ GRASP reconstruction}

Ten parameters with VS-GR reconstruction showed significant differences between responders and nonresponders, which including volume transfer constant (Ktrans) max, Ktrans mean, Ktrans 75\%, intravasation rate contrast (Kep) max, extravascular extracellular volume fraction $(\mathrm{Ve})$ mean, Ve $75 \%$, Vp max, the initial area-under-the- concentration versus time curve (AUC) max, AUC mean, AUC 75\%. 13 parameters with GRASP reconstruction showed significant differences between

Table 3 Differences among TRG1-5 for DCE-MRI parameters with VS-GR and GRASP reconstruction

\begin{tabular}{|c|c|c|c|c|c|c|c|c|c|c|c|c|c|c|}
\hline \multirow[t]{2}{*}{ parameters } & \multicolumn{7}{|c|}{ VS-GR reconstruction } & \multicolumn{7}{|c|}{ GRASP reconstruction } \\
\hline & $\overline{\mathrm{TRG} 1}$ & TRG2 & TRG3 & TRG4 & TRG5 & $x^{2}$ & $\begin{array}{l}P \\
\text { value }\end{array}$ & TRG1 & TRG2 & TRG3 & TRG4 & TRG5 & $x^{2}$ & $\begin{array}{l}P \\
\text { value }\end{array}$ \\
\hline Ktrans max & $\begin{array}{l}0.000 \\
(0.000 \\
0.099)\end{array}$ & $\begin{array}{l}1.075 \\
(0.630 \\
1.643)\end{array}$ & $\begin{array}{l}0.713 \\
(0.553 \\
1.405)\end{array}$ & $\begin{array}{l}2.477 \\
(1.800 \\
5.000)\end{array}$ & $\begin{array}{l}2.396 \\
(1.357 \\
3.420)\end{array}$ & 20.101 & $\begin{array}{l}< \\
0.001\end{array}$ & $\begin{array}{l}0.000 \\
(0.000 \\
0.086)\end{array}$ & $\begin{array}{l}0.159 \\
(0.100 \\
0.304)\end{array}$ & $\begin{array}{l}0.170 \\
(0.117 \\
0.207)\end{array}$ & $\begin{array}{l}0.324 \\
(0.198 \\
0.905)\end{array}$ & $\begin{array}{l}0.310 \\
(0.200 \\
0.424)\end{array}$ & 15.533 & 0.004 \\
\hline $\begin{array}{l}\text { Ktrans } \\
\text { mean }\end{array}$ & $\begin{array}{l}0.000 \\
(0.000 \\
0.037)\end{array}$ & $\begin{array}{l}0.277 \\
(0.105 \\
0.343)\end{array}$ & $\begin{array}{l}0.189 \\
(0.094 \\
0.369)\end{array}$ & $\begin{array}{l}0.239 \\
(0.200 \\
0.549)\end{array}$ & $\begin{array}{l}0.315 \\
(0.166 \\
0.405)\end{array}$ & 12.368 & 0.015 & $\begin{array}{l}0.000 \\
(0.000 \\
0.027)\end{array}$ & $\begin{array}{l}0.055 \\
(0.030 \\
0.106)\end{array}$ & $\begin{array}{l}0.044 \\
(0.025 \\
0.068)\end{array}$ & $\begin{array}{l}0.060 \\
(0.045 \\
0.126)\end{array}$ & $\begin{array}{l}0.074 \\
(0.051 \\
0.095)\end{array}$ & 13.432 & 0.009 \\
\hline Ktrans 75\% & $\begin{array}{l}0.000 \\
(0.000 \\
0.052)\end{array}$ & $\begin{array}{l}0.359 \\
(0.123 \\
0.467)\end{array}$ & $\begin{array}{l}0.307 \\
(0.123 \\
0.491)\end{array}$ & $\begin{array}{l}0.372 \\
(0.271 \\
0.572)\end{array}$ & $\begin{array}{l}0.422 \\
(0.200 \\
0.562)\end{array}$ & 12.313 & 0.015 & $\begin{array}{l}0.000 \\
(0.000 \\
0.034)\end{array}$ & $\begin{array}{l}0.082 \\
(0.043 \\
0.129)\end{array}$ & $\begin{array}{l}0.056 \\
(0.033 \\
0.082)\end{array}$ & $\begin{array}{l}0.074 \\
(0.060 \\
0.160)\end{array}$ & $\begin{array}{l}0.091 \\
(0.065 \\
0.122)\end{array}$ & 12.531 & 0.014 \\
\hline Kep max & $\begin{array}{l}0.000 \\
(0.000 \\
0.200)\end{array}$ & $\begin{array}{l}3.131 \\
(2.127 \\
5.898)\end{array}$ & $\begin{array}{l}2.208 \\
(1.657 \\
3.024)\end{array}$ & $\begin{array}{l}4.506 \\
(2.729 \\
7.424)\end{array}$ & $\begin{array}{l}4.868 \\
(2.823 \\
6.578)\end{array}$ & 16.684 & 0.002 & $\begin{array}{l}0.000 \\
(0.000 \\
0.489)\end{array}$ & $\begin{array}{l}0.810 \\
(0.543 \\
1.410)\end{array}$ & $\begin{array}{l}0.929 \\
(0.580 \\
1.121)\end{array}$ & $\begin{array}{l}1.543 \\
(1.010 \\
1.985)\end{array}$ & $\begin{array}{l}1.390 \\
(0.854 \\
1.877)\end{array}$ & 14.455 & 0.006 \\
\hline Kep mean & $\begin{array}{l}0.000 \\
(0.000 \\
0.002)\end{array}$ & $\begin{array}{l}0.723 \\
(0.388 \\
0.824)\end{array}$ & $\begin{array}{l}0.215 \\
(0.113 \\
0.493)\end{array}$ & $\begin{array}{l}0.349 \\
(0.252 \\
1.185)\end{array}$ & $\begin{array}{l}0.537 \\
(0.361 \\
0.844)\end{array}$ & 15.088 & 0.005 & $\begin{array}{l}0.000 \\
(0.000 \\
0.123)\end{array}$ & $\begin{array}{l}0.294 \\
(0.167 \\
0.558)\end{array}$ & $\begin{array}{l}0.212 \\
(0.167 \\
0.455)\end{array}$ & $\begin{array}{l}0.272 \\
(0.213 \\
0.434)\end{array}$ & $\begin{array}{l}0.331 \\
(0.227 \\
0.432)\end{array}$ & 10.358 & 0.035 \\
\hline Kep 75\% & $\begin{array}{l}0.000 \\
(0.000 \\
0.005)\end{array}$ & $\begin{array}{l}1082 \\
(0.833 \\
1.568)\end{array}$ & $\begin{array}{l}0.361 \\
(0.010 \\
0.799)\end{array}$ & $\begin{array}{l}0.539 \\
(0.327 \\
1.571)\end{array}$ & $\begin{array}{l}0.897 \\
(0.545 \\
1.361)\end{array}$ & 15.254 & 0.004 & $\begin{array}{l}0.000 \\
(0.000 \\
0.160)\end{array}$ & $\begin{array}{l}0.472 \\
(0.262 \\
0.664)\end{array}$ & $\begin{array}{l}0.277 \\
(0.228 \\
0.532)\end{array}$ & $\begin{array}{l}0.358 \\
(0.274 \\
0.552)\end{array}$ & $\begin{array}{l}0.451 \\
(0.282 \\
0.540)\end{array}$ & 11.033 & 0.026 \\
\hline Ve max & $\begin{array}{l}1.000 \\
(0.510 \\
1.000)\end{array}$ & $\begin{array}{l}1.000 \\
(1.000 \\
1.000)\end{array}$ & $\begin{array}{l}1.000 \\
(1.000 \\
1.000)\end{array}$ & $\begin{array}{l}1.000 \\
(1.000 \\
1.000)\end{array}$ & $\begin{array}{l}1.000 \\
(1.000 \\
1.000)\end{array}$ & 7.180 & 0.127 & $\begin{array}{l}1.000 \\
(1.000 \\
1.000)\end{array}$ & $\begin{array}{l}0.824 \\
(0.376 \\
1.000)\end{array}$ & $\begin{array}{l}1.000 \\
(0.417 \\
1.000)\end{array}$ & $\begin{array}{l}1.000 \\
(1.000 \\
1.000)\end{array}$ & $\begin{array}{l}1.000 \\
(0.826 \\
1.000)\end{array}$ & 3.447 & 0.486 \\
\hline Ve mean & $\begin{array}{l}0.000 \\
(0.000 \\
0.002)\end{array}$ & $\begin{array}{l}\text { O.349 } \\
(0.111 \\
0.438)\end{array}$ & $\begin{array}{l}0.369 \\
(0.139 \\
0.434)\end{array}$ & $\begin{array}{l}0.331 \\
(0.256 \\
0.386)\end{array}$ & $\begin{array}{l}0.317 \\
(0.203 \\
0.359)\end{array}$ & 11.552 & 0.021 & $\begin{array}{l}0.000 \\
(0.000 \\
0.200)\end{array}$ & $\begin{array}{l}0.196 \\
(0.150 \\
0.207)\end{array}$ & $\begin{array}{l}0.189 \\
(0.159 \\
0.275)\end{array}$ & $\begin{array}{l}0.230 \\
(0.199 \\
0.303)\end{array}$ & $\begin{array}{l}0.230 \\
(0.199 \\
0.287)\end{array}$ & 9.809 & 0.044 \\
\hline Ve $75 \%$ & $\begin{array}{l}0.000 \\
(0.000 \\
0.0007)\end{array}$ & $\begin{array}{l}0.387 \\
(0.064 \\
0.555)\end{array}$ & $\begin{array}{l}0.505 \\
(0.001 \\
0.604)\end{array}$ & $\begin{array}{l}0.503 \\
(0.373 \\
0.570)\end{array}$ & $\begin{array}{l}0.468 \\
(0.303 \\
0.508)\end{array}$ & 11.478 & 0.022 & $\begin{array}{l}0.000 \\
(0.000 \\
0.214)\end{array}$ & $\begin{array}{l}0.220 \\
(0.175 \\
0.250)\end{array}$ & $\begin{array}{l}0.219 \\
(0.180 \\
0.305)\end{array}$ & $\begin{array}{l}0.257 \\
(0.163 \\
0.311)\end{array}$ & $\begin{array}{l}0.256 \\
(0.219, \\
0.328)\end{array}$ & 8.518 & 0.074 \\
\hline Vp max & $\begin{array}{l}0.000 \\
(0.000 \\
0.007)\end{array}$ & $\begin{array}{l}0.067 \\
(0.033 \\
0.115)\end{array}$ & $\begin{array}{l}0.058 \\
(0.041 \\
0.101)\end{array}$ & $\begin{array}{l}0.244 \\
(0.056 \\
0.630)\end{array}$ & $\begin{array}{l}0.143 \\
(0.073 \\
0.249)\end{array}$ & 14.198 & 0.007 & $\begin{array}{l}0.000 \\
(0.000 \\
0.034)\end{array}$ & $\begin{array}{l}0.066 \\
(0.050 \\
0.104)\end{array}$ & $\begin{array}{l}0.082 \\
(0.045 \\
0.137)\end{array}$ & $\begin{array}{l}0.205 \\
(0.103 \\
0.335)\end{array}$ & $\begin{array}{l}0.134 \\
(0.090 \\
0.236)\end{array}$ & 16.581 & 0.002 \\
\hline Vp mean & $\begin{array}{l}0.000 \\
(0.000 \\
0.0001)\end{array}$ & $\begin{array}{l}0.004 \\
(0.001 \\
0.007)\end{array}$ & $\begin{array}{l}0.001 \\
(0.001 \\
0.007)\end{array}$ & $\begin{array}{l}0.003 \\
(0.000 \\
0.009)\end{array}$ & $\begin{array}{l}0.002 \\
(0.000 \\
0.005)\end{array}$ & 9.772 & 0.044 & $\begin{array}{l}0.000 \\
(0.000 \\
0.001)\end{array}$ & $\begin{array}{l}0.009 \\
(0.003 \\
0.015)\end{array}$ & $\begin{array}{l}0.003 \\
(0.001 \\
0.027)\end{array}$ & $\begin{array}{l}0.019 \\
(0.006 \\
0.063)\end{array}$ & $\begin{array}{l}0.014 \\
(0.009 \\
0.016)\end{array}$ & 17.041 & 0.002 \\
\hline Vp 75\% & $\begin{array}{l}0.000 \\
(0.000 \\
0.0007)\end{array}$ & $\begin{array}{l}0.001 \\
(0.001 \\
0.004)\end{array}$ & $\begin{array}{l}0.001 \\
(0.001 \\
0.006)\end{array}$ & $\begin{array}{l}0.001 \\
(0.001 \\
0.001)\end{array}$ & $\begin{array}{l}0.001 \\
(0.001 \\
0.001)\end{array}$ & 9.602 & 0.048 & $\begin{array}{l}0.000 \\
(0.000 \\
0008)\end{array}$ & $\begin{array}{l}0.011 \\
(0.003 \\
0.022)\end{array}$ & $\begin{array}{l}0.001 \\
(0.001 \\
0.041)\end{array}$ & $\begin{array}{l}0.033 \\
(0.007 \\
0.080)\end{array}$ & $\begin{array}{l}0.023 \\
(0.014, \\
0.038)\end{array}$ & 17.523 & 0.002 \\
\hline AUC max & $\begin{array}{l}0.000 \\
(0.000 \\
0.316)\end{array}$ & $\begin{array}{l}0.071 \\
(0.059 \\
0.089)\end{array}$ & $\begin{array}{l}0.083 \\
(0.070 \\
0.089)\end{array}$ & $\begin{array}{l}0.101 \\
(0.090 \\
0.119)\end{array}$ & $\begin{array}{l}0.121 \\
(0.089 \\
0.147)\end{array}$ & 21.365 & $\begin{array}{l}< \\
0.001\end{array}$ & $\begin{array}{l}0.000 \\
(0.000 \\
1.619)\end{array}$ & $\begin{array}{l}1.738 \\
(0.974 \\
2.350)\end{array}$ & $\begin{array}{l}3.344 \\
(2.259 \\
6.248)\end{array}$ & $\begin{array}{l}4.561 \\
(2.326 \\
6.424)\end{array}$ & $\begin{array}{l}3.364 \\
(2.516 \\
4.512)\end{array}$ & 16.454 & 0.002 \\
\hline AUC mean & $\begin{array}{l}0.000 \\
(0.000 \\
0.012)\end{array}$ & $\begin{array}{l}0.036 \\
(0.018 \\
0.045)\end{array}$ & $\begin{array}{l}0.043 \\
(0.026 \\
0.045)\end{array}$ & $\begin{array}{l}0.369 \\
(0.032 \\
0.041)\end{array}$ & $\begin{array}{l}0.040 \\
(0.029 \\
0.047)\end{array}$ & 11.445 & 0.022 & $\begin{array}{l}0.000 \\
(0.000 \\
0.793)\end{array}$ & $\begin{array}{l}0.940 \\
(0.562, \\
1.469)\end{array}$ & $\begin{array}{l}1.473 \\
(1.036 \\
1.617)\end{array}$ & $\begin{array}{l}1.688 \\
(1.035 \\
2.375)\end{array}$ & $\begin{array}{l}1.538 \\
(1.190 \\
1.875)\end{array}$ & 12.605 & 0.013 \\
\hline AUC 75\% & $\begin{array}{l}0.000 \\
(0.000 \\
0.015)\end{array}$ & $\begin{array}{l}0.045 \\
(0.025 \\
0.056)\end{array}$ & $\begin{array}{l}0.052 \\
(0.034, \\
0.054)\end{array}$ & $\begin{array}{l}0.047 \\
(0.041 \\
0.056)\end{array}$ & $\begin{array}{l}0.053 \\
(0.038 \\
0.061)\end{array}$ & 11.980 & 0.018 & $\begin{array}{l}0.000 \\
(0.000 \\
0.965)\end{array}$ & $\begin{array}{l}1.180 \\
(0.734 \\
1.661)\end{array}$ & $\begin{array}{l}1.642 \\
(1.232, \\
1.981)\end{array}$ & $\begin{array}{l}2.041 \\
(1.234 \\
2.748)\end{array}$ & $\begin{array}{l}1.854 \\
(1.417 \\
2.149)\end{array}$ & 12.994 & 0.011 \\
\hline
\end{tabular}

Note. -Data are median (P25, P75) 
Table 4 Differences between responder and non-responder groups for DCE-MRI parameters with VS-GR/GRASP reconstruction

\begin{tabular}{|c|c|c|c|c|c|c|c|c|}
\hline \multirow[t]{2}{*}{ parameters } & \multicolumn{4}{|l|}{ VS-GR reconstruction } & \multicolumn{4}{|c|}{ GRASP reconstruction } \\
\hline & responder & non-responder & U value & $P$ value & responder & non-responder & U value & $P$ value \\
\hline Ktrans max & $0.314(0.000,1.097)$ & $2.303(1.172,3.564)$ & 51.0 & $<0.001$ & $0.101(0.000,0.189)$ & $0.304(0.196,0.415)$ & 69.0 & 0.002 \\
\hline Ktrans mean & $0.055(0.000,0.298)$ & $0.299(0.157,0.387)$ & 92.0 & 0.007 & $0.031(0.000,0.067)$ & $0.065(0.046,0.094)$ & 104.0 & 0.015 \\
\hline Ktrans 75\% & $0.069(0.000,0.395)$ & $0.404(0.200,0.545)$ & 92.0 & 0.007 & $0.043(0.000,0.097)$ & $0.082(0.061,0.121)$ & 115.0 & 0.027 \\
\hline Kep max & $1.088(0.000,3.309)$ & $4.272(2.307,6.527)$ & 90.0 & 0.007 & $0.559(0.000,0.934)$ & $1.342(0.861,1.875)$ & 75.0 & 0.002 \\
\hline Kep mean & $0.149(0.000,0.751)$ & $0.506(0.251,0.823)$ & 140.0 & 0.088 & $0.160(0.000,0.340)$ & $0.318(0.212,0.432)$ & 117.0 & 0.030 \\
\hline Kep 75\% & $0.384(0.000,1.095)$ & $0.772(0.363,1.334)$ & 154.0 & 0.155 & $0.215(0.000,0.509)$ & $0.429(0.268,0.536)$ & 137.0 & 0.077 \\
\hline Ve max & $1.000(1.000,1.000)$ & $1.000(1.000,1.000)$ & 199.5 & 0.099 & $1.000(0.736,1.000)$ & $1.000(0.875,1.000)$ & 223.0 & 0.979 \\
\hline Ve mean & $0.030(0.000,0.388)$ & $0.323(0.201,0.370)$ & 124.0 & 0.042 & $0.163(0.000,0.207)$ & $0.227(0.189,0.276)$ & 93.0 & 0.008 \\
\hline Ve $75 \%$ & $0.006(0.000,0.471)$ & $0.475(0.297,0.521)$ & 112.0 & 0.023 & $0.187(0.000,0.250)$ & $0.250(0.209,0.311)$ & 104.0 & 0.015 \\
\hline Vp max & $0.017(0.000,0.068)$ & $0.140(0.056,0.316)$ & 71.0 & 0.002 & $0.046(0.000,0.070)$ & $0.133(0.085,0.233)$ & 63.0 & 0.001 \\
\hline Vp mean & $0.0004(0.0000,0.0048)$ & $0.002(0.001,0.006)$ & 135.0 & 0.071 & $0.001(0.000,0.009)$ & $0.014(0.008,0.030)$ & 67.0 & 0.001 \\
\hline Vp 75\% & $0.001(0.001,0.001)$ & $0.001(0.001,0.001)$ & 142.0 & 0.096 & $0.001(0.000,0.012)$ & $0.023(0.008,0.042)$ & 65.0 & 0.001 \\
\hline AUC max & $0.050(0.000,0.076)$ & $0.108(0.085,0.137)$ & 40.0 & $<0.001$ & $1.062(0.000,2.220)$ & $3.424(2.489,4.832)$ & 31.0 & $<0.001$ \\
\hline AUC mean & $0.016(0.000,0.040)$ & $0.039(0.030,0.047)$ & 90.0 & 0.007 & $0.569(0.000,1.236)$ & $1.515(1.188,1.901)$ & 60.0 & 0.001 \\
\hline AUC 75\% & $0.020(0.000,0.049)$ & $0.052(0.052,0.060)$ & 86.0 & 0.005 & $0.753(0.000,1.497)$ & $1.854(1.403,2.203)$ & 55.0 & 0.001 \\
\hline
\end{tabular}

Note. - Data are median (P25, P75)

responders and non-responders, which including Ktrans max, Ktrans mean, Ktrans 75\%, Kep max, Kep mean, Ve mean, Ve 75\%, Vp max, Vp mean, Vp 75\%, AUC max, AUC mean, AUC 75\% (Table 4).

\section{Correlation between parameters with VS-GR/GRASP reconstruction and TRG/response}

With VS-GR, 10/15 parameters significantly correlated with TRG and response groups. Of these, only AUCmax showed moderate correlation with TRG, 7 showed low correlation and 2 showed negligible correlation with TRG. 8 showed low correlation and 2 showed negligible correlation with response groups. With GRASP, 13/15 parameters significantly correlated with TRG and response groups. Of these, 10 showed low correlation and 3 showed negligible correlation with TRG. 11 showed low correlation and 2 showed negligible correlation with TRGs (Table 5).

Table 5 DCE-MRI parameters with VS-GR/GRASP stratified according to TRGs and response

\begin{tabular}{|c|c|c|c|c|}
\hline \multirow[t]{3}{*}{ Parameters } & \multicolumn{2}{|l|}{ TRG1-5 } & \multicolumn{2}{|c|}{ responder and non-responder } \\
\hline & $\overline{V S-G R}$ & GRASP & VS-GR & GRASP \\
\hline & $r^{*}(P)$ & $r^{*}(P)$ & $r^{*}(P)$ & $r^{*}(P)$ \\
\hline Ktrans max & $0.409(0.001)$ & $0.343(0.006)$ & $0.443(<0.001)$ & $0.396(0.001)$ \\
\hline Ktrans mean & $0.305(0.014)$ & $0.320(0.010)$ & $0.338(0.006)$ & $0.307(0.014)$ \\
\hline Ktrans $75 \%$ & $0.318(0.011)$ & $0.282(0.024)$ & $0.338(0.006)$ & $0.279(0.026)$ \\
\hline Kep max & $0.379(0.002)$ & $0.323(0.009)$ & $0.343(0.006)$ & $0.381(0.002)$ \\
\hline Kep mean & $0.314(0.012)$ & $0.255(0.042)$ & $0.215(0.088)$ & $0.274(0.029)$ \\
\hline Kep 75\% & $0.283(0.023)$ & $0.238(0.058)$ & $0.179(0.157)$ & $0.223(0.077)$ \\
\hline Ve max & $0.097(0.446)$ & $-0.035(0.784)$ & $0.208(0.099)$ & $-0.003(0.979)$ \\
\hline Ve mean & $0.125(0.324)$ & $0.318(0.010)$ & $0.256(0.041)$ & $0.335(0.007)$ \\
\hline Ve $75 \%$ & $0.151(0.234)$ & $0.330(0.008)$ & $0.286(0.022)$ & $0.307(0.014)$ \\
\hline Vp max & $0.312(0.012)$ & $0.333(0.007)$ & $0.391(0.001)$ & $0.412(0.001)$ \\
\hline Vp mean & $0.158(0.213)$ & $0.371(0.003)$ & $0.228(0.070)$ & $0.402(0.001)$ \\
\hline Vp 75\% & $0.115(0.366)$ & $0.370(0.003)$ & $0.210(0.096)$ & $0.407(0.001)$ \\
\hline AUC max & $0.524(<0.001)$ & $0.253(0.044)$ & $0.471(<0.001)$ & $0.494(<0.001)$ \\
\hline AUC mean & $0.294(0.018)$ & $0.306(0.014)$ & $0.343(0.006)$ & $0.419(0.001)$ \\
\hline AUC 75\% & $0.314(0.012)$ & $0.307(0.014)$ & $0.353(0.004)$ & $0.432(<0.001)$ \\
\hline
\end{tabular}


Diagnostic performance of DCE-MRI parameters with VSGR/ GRASP reconstruction between responder and nonresponder groups

Seven parameters with VS-GR/GRASP reconstruction showed good or excellent diagnostic performance between responders and non-responders, which including Ktrans max, Ktrans mean, Kep max, Vp max, AUC max, AUC mean, AUC 75\%. In general, the seven variables had similar diagnostic performance in the two reconstructions. Among the seven variables, AUC max showed excellent performance in response groups (AUC $>0.90, P<0.05)$ (Table 6).

\section{Discussion}

This study demonstrated that GRASP reconstruction may affect the results of DCE-MRI, DCE-MRI with VSGR and GRASP reconstruction could assess tumor response, and pharmacokinetic parameters with GRASP and VS-GR reconstruction may help stratify responders from non-responders in patients with EC treated by $\mathrm{nCT}$. In this study, 10 post-nCT pharmacokinetic parameters with VS-GR reconstruction and 13 parameters with GRASP reconstruction showed statistically significant differences between responders and nonresponders. Moreover, GRASP reconstruction provided more parameters than VS-GR reconstruction. However, seven parameters with VS-GR/GRASP reconstruction showed good or excellent diagnostic performance between responders and non-responders and no significant difference in diagnostic performance between VS-GR and GRASP reconstructions.

Table 6 Diagnostic performance of DCE-MRI parameters with VS-GR/GRASP according to response groups

\begin{tabular}{|c|c|c|c|c|c|c|c|}
\hline \multirow[t]{2}{*}{ Parameters } & \multicolumn{2}{|c|}{ Sensitivity (\%) } & \multicolumn{2}{|c|}{ Speciticity (\%) } & \multicolumn{2}{|l|}{$A \cup C^{*}$} & \multirow[t]{2}{*}{$P$} \\
\hline & VS-GR & GRASP & VS-GR & GRASP & VS-GR & GRASP & \\
\hline Ktrans max & 87.5 & 75.0 & 76.8 & 85.7 & 0.886 & 0.846 & 0.464 \\
\hline Ktrans mean & 62.5 & 75.0 & 98.2 & 83.9 & 0.795 & 0.768 & 0.634 \\
\hline Ktrans 75\% & 62.5 & 75.0 & 98.2 & 78.6 & 0.795 & 0.743 & 0.256 \\
\hline Kep max & 50.0 & 75.0 & 100.0 & 83.9 & 0.799 & 0.833 & 0.708 \\
\hline Kep mean & 50.0 & 75.0 & 100.0 & 76.8 & 0.687 & 0.739 & 0.566 \\
\hline Kep 75\% & 50.0 & 62.5 & 100.0 & 87.5 & 0.656 & 0.694 & 0.658 \\
\hline Ve max & 12.5 & 87.5 & 100.0 & 3.6 & 0.555 & 0.502 & 0.604 \\
\hline Ve mean & 62.5 & 87.5 & 98.2 & 64.3 & 0.723 & 0.792 & 0.672 \\
\hline Ve $75 \%$ & 75.0 & 75.0 & 78.6 & 67.9 & 0.750 & 0.768 & 0.905 \\
\hline Vp max & 87.5 & 87.5 & 71.4 & 78.6 & 0.842 & 0.859 & 0.785 \\
\hline Vp mean & 62.5 & 62.5 & 85.7 & 96.4 & 0.699 & 0.850 & 0.221 \\
\hline Vp 75\% & 62.5 & 62.5 & 82.1 & 98.2 & 0.683 & 0.855 & 0.211 \\
\hline AUC max & 87.5 & 100.0 & 82.1 & 80.4 & 0.911 & 0.931 & 0.580 \\
\hline AUC mean & 62.5 & 62.5 & 96.4 & 98.2 & 0.799 & 0.866 & 0.249 \\
\hline AUC 75\% & 62.5 & 62.5 & 96.4 & 98.2 & 0.808 & 0.877 & 0.280 \\
\hline
\end{tabular}

Most DCE-MRI studies only analyzed parts of parameters, such as Ktrans mean, kep mean, Ve mean, and AUC, and showed DCE-MRI could assess the response to therapy [22]. In the current study, we tried to analyze more parameters acquired from DCE-MRI, and 15 parameters were analyzed.

It was reported that DCE-MRI with GRASP reconstruction could provide near-isotropic resolution and higher in-plane spatial resolution [13]. Contribution to the VS-GR images with a $2.1 \mathrm{~s}$ apparent temporal resolution is from $\mathrm{a} \sim 21 \mathrm{~s}$ time footprint acquisition, while GRASP is reconstructed from a $4.5 \mathrm{~s}$ time footprint, higher temporal resolution normally leads to an improved AIF, which is used for more accurate pharmacokinetics parameters calculation [23]. Compared to conventional VS-GR DCE-MRI, this could result in better acquisition of pharmacokinetic parameters potentially which has been reported in hepatocellular carcinoma, renal cell carcinoma and rectal cancer [13] [16] [15]. VS-GR DCE-MRI had been used in EC [12], however, GRASP reconstruction has not been reported to be compared with VS-GR reconstruction in EC. The AIF plays an important role for the pharmacokinetic models in determining the quantitative measurements of physiological parameters, where small differences in AIF may lead to large differences in quantitative maps and higher temporal resolution gives smaller differences.

More parameters with GRASP showed significant correlation with TRGs and response groups than those with VS-GR reconstruction. Both 10/15 parameters with VSGR reconstruction showed significant correlation with TRGs and response groups, and both 13/15 parameters with GRASP reconstruction showed significant correlation with TRGs and response groups. It may be the effect of GRASP reconstruction, providing higher time resolution and more information.

It is critical to detecting residual cancer post-nT. Fortunately, some pharmacokinetic parameters between TGRs showed significant differences in this study. The information of whole tumor, rather than a single axial level, was assessed in our study, which theoretically provides a more comprehensive representation of tumor information than that provided by a single-level analysis.

FDG-PET have been used for neoadjuvant treatment response assessment in EC [24], and the FDG-PET response after neoadjuvant treatment could predict the pathological response and seems to be related to survival [25-27]. However, Van Rossum et al. showed that accuracy of imaging is insufficient in predicting pathologic response [28], and the prognostic value of FDG-PET response after chemoradiotherapy has not been definitively established $[29,30]$.

There were several limitations in this study. First, one critical step in quantifying DCE MRI parameters is to 
sample AIF from a major artery. However, to sample AIF in esophageal images can be challenging, because of its small size. Li et al. showed automatically sampling AIF by utilizing temporal and spatial features in a multistep interleaved manner, that highly resembled those manually sampled ones in lower extremity arteries [31]. Second, limited sample size, the number of TRG1 in particular, may lead to bias. Finally, GRASP reconstruction required offline reconstruction, more computing ability and more time for reconstruction.

\section{Conclusions}

Several pharmacokinetic parameters of DCE-MRI reconstructed by GRASP and VS-GR show significant differences between TRGs and response groups and thus can be used to non-invasively predict tumor response. GRASP reconstruction provided more parameters than VS-GR reconstruction, which maybe showed additionally significant merit, and larger sample size study need to assess it furtherly.

\section{Abbreviations \\ AIF: Arterial input function; AUC: Area under the ROC curve; AUC: the initial area-under-the- concentration versus time curve; DCE-MRI: Dynamic contrast- enhanced Magnetic resonance imaging; EC: Esophageal cancer; GRASP: Golden-angle radial sparse parallel; Kep: Rate contrast; Ktrans: Volume transfer constant; nCT: Neoadjuvant chemotherapy; ROC: Receiver operating characteristic; ROI: Regions of interest; TRG: Tumor Regression Grade; Ve: Extravascular extracellular volume fraction; Vp: Plasma volume fraction; VS-GR: View-sharing with golden-angle radial profile}

\section{Acknowledgements}

Not applicable.

\begin{abstract}
Authors' contributions
Guarantors of integrity of entire study, JQu.; study concepts/study design or data acquisition or data analysis/interpretation, all authors; manuscript drafting or manuscript revision for important intellectual content, all authors; manuscript final version approval, all authors; agrees to ensure any questions related to the work are appropriately resolved, all authors; literature research, YL., LM., JQin., JQu.; clinical studies, YL., LM., ZW., JG., HZ., XY., HL., JQin, JQu.; statistical analysis, YZhao.; and manuscript editing, YL., LM., JQin, JQu, IK. All authors have read and approved the final manuscript.
\end{abstract}

\section{Funding}

This study has received funding by the General Programs of the National Natural Science Foundation of China (No.81972802), the Project of Henan Health of China (No. 201203149), and Special Funding of the Henan Health Science and Technology Innovation Talent Project (No.

201004057) JRQ is the principle Investigator. The funders had no role in study design, data collection and analysis, decision to publish, or preparation of the manuscript.

\section{Availability of data and materials}

The datasets used and/or analyzed during the current study are available from the corresponding author on reasonable request.

\section{Ethics approval and consent to participate}

The study protocol was approved by institutional review board of Henan Cancer Hospital, and written informed consent was obtained from all participants.

\section{Consent for publication}

Not applicable.

\section{Competing interests}

The authors declare that they have no competing interests.

\section{Author details}

${ }^{1}$ Department of Radiology, the Affiliated Cancer Hospital of Zhengzhou University \& Henan Cancer Hospital, 127 Dongming Road, Zhengzhou 450008, Henan, China. ${ }^{2}$ Advanced Application team, GE Healthcare, 1 Hua Tuo Road, Zhangjiang Hi-tech park, Pudong, Shanghai 201203, China. ${ }^{3}$ Department of Thoracic Surgery, the Affiliated Cancer Hospital of Zhengzhou University \& Henan Cancer Hospital, 127 Dongming Road, Zhengzhou 450008, Henan, China. ${ }^{4}$ National Cancer Center/Cancer Hospital, Chinese Academy of Medical Sciences and Peking Union Medical College, Beijing 100021, China. ${ }^{5}$ NEA MR Collaboration, Siemens Ltd, 278, Zhouzhu Road, Pudong New Area, Shanghai 201318, China. ${ }^{6}$ Department of Radiology, Johns Hopkins University School of Medicine, Baltimore, MD 21205-2196, USA.

Received: 24 June 2019 Accepted: 3 October 2019

Published online: 24 October 2019

\section{References}

1. Heethuis SE, van Rossum PS, Lips IM, Goense L, Voncken FE, Reerink O, van Hillegersberg R, Ruurda JP, Philippens ME, van Vulpen M, et al. Dynamic contrast-enhanced MRI for treatment response assessment in patients with oesophageal cancer receiving neoadjuvant chemoradiotherapy. Radiother Oncol. 2016:120(1):128-35.

2. Watanabe M, Mine S, Nishida K, Yamada K, Shigaki H, Matsumoto A, Sano T. Salvage Esophagectomy after definitive Chemoradiotherapy for patients with esophageal squamous cell carcinoma: who really benefits from this high-risk surgery? Ann Surg Oncol. 2015;22(13):4438-44.

3. Kiyozumi Y, Yoshida N, Ishimoto T, Yagi T, Koga Y, Uchihara T, Sawayama H, Hiyoshi $Y$, Iwatsuki M, Baba $Y$, et al. Prognostic factors of salvage Esophagectomy for residual or recurrent esophageal squamous cell carcinoma after definitive Chemoradiotherapy. World J Surg. 2018;42(9): 2887-93.

4. Pinto E, Cavallin F, Alfieri R, Saadeh LM, Mantoan S, Cagol M, Castoro C, Scarpa M. Impact of esophagectomy for cancer on patients' occupational status. Eur J Surg Oncol. 2016;42(1):103-9.

5. Committee for Scientific Affairs TJAfTS, Masuda M, Kuwano H, Okumura M, Arai H, Endo S, Doki Y, Kobayashi J, Motomura N, Nishida H, et al. Thoracic and cardiovascular surgery in Japan during 2013: Annual report by the Japanese Association for Thoracic Surgery. Gen Thorac Cardiovasc Surg. 2015;63(12):670-701

6. Raymond DP, Seder CW, Wright CD, Magee MJ, Kosinski AS, Cassivi SD, Grogan EL, Blackmon SH, Allen MS, Park BJ, et al. Predictors of major morbidity or mortality after resection for esophageal Cancer: a Society of Thoracic Surgeons general thoracic surgery database risk adjustment model. Ann Thorac Surg. 2016;102(1):207-14.

7. Nakajima M, Muroi H, Kikuchi M, Takahashi M, Ihara K, Shida Y, Kurayama E, Ogata H, Yamaguchi S, Sasaki K, et al. Adverse prognostic factors of advanced esophageal Cancer in patients undergoing induction therapy with Docetaxel, Cisplatin and 5-fluorouracil. Anticancer Res. 2018;38(2):911-8.

8. Ando N, Kato H, Igaki H, Shinoda M, Ozawa S, Shimizu H, Nakamura T, Yabusaki H, Aoyama N, Kurita A, et al. A randomized trial comparing postoperative adjuvant chemotherapy with cisplatin and 5-fluorouracil versus preoperative chemotherapy for localized advanced squamous cell carcinoma of the thoracic esophagus (JCOG9907). Ann Surg Oncol. 2012; 19(1):68-74.

9. Blazeby JM, Sanford E, Falk SJ, Alderson D, Donovan JL. Health-related quality of life during neoadjuvant treatment and surgery for localized esophageal carcinoma. Cancer. 2005;103(9):1791-9.

10. Xi M, Liao Z, Hofstetter WL, Komaki R, Ho L, Lin SH. (18) F-FDG PET response after induction chemotherapy can predict who will benefit from subsequent Esophagectomy after Chemoradiotherapy for esophageal adenocarcinoma. J Nucl Med. 2017;58(11):1756-63.

11. Oberholzer K, Pohlmann A, Schreiber W, Mildenberger P, Kunz P, Schmidberger $\mathrm{H}$, Junginger $\mathrm{T}$, Duber $\mathrm{C}$. Assessment of tumor microcirculation with dynamic contrast-enhanced MRI in patients with esophageal cancer: initial experience. J Magn Reson Imaging. 2008:27(6): 1296-301. 
12. Lei J, Han Q, Zhu S, Shi D, Dou S, Su Z, Xu X. Assessment of esophageal carcinoma undergoing concurrent chemoradiotherapy with quantitative dynamic contrast-enhanced magnetic resonance imaging. Oncol Lett. 2015; 10(6):3607-12

13. Chandarana H, Feng L, Block TK, Rosenkrantz AB, Lim RP, Babb JS, Sodickson DK, Otazo R. Free-breathing contrast-enhanced multiphase MRI of the liver using a combination of compressed sensing, parallel imaging, and goldenangle radial sampling. Investig Radiol. 2013;48(1):10-6.

14. Braren R, Curcic J, Remmele S, Altomonte J, Ebert O, Rummeny EJ, Steingoetter A. Free-breathing quantitative dynamic contrast-enhanced magnetic resonance imaging in a rat liver tumor model using dynamic radial T (1) mapping. Investig Radiol. 2011;46(10):624-31.

15. Attenberger UI, Liu J, Riffel P, Budjan J, Grimm R, Block KT, Schoenberg SO Wang X, Hausmann D. Quantitative perfusion analysis of the rectum using Golden-angle radial sparse parallel magnetic resonance imaging: initial experience and comparison to time-resolved angiography with interleaved stochastic trajectories. Investig Radiol. 2017;52(12):715-24

16. Riffel P, Zoellner FG, Budjan J, Grimm R, Block TK, Schoenberg SO, Hausmann D. "one-stop shop": free-breathing dynamic contrast-enhanced magnetic resonance imaging of the kidney using iterative reconstruction and continuous Golden-angle radial sampling. Investig Radiol. 2016;51(11):714-9.

17. Yanan L, Ling M, Zhaoqi W, Jia G, Hongkai Z, Xu Y, Hui L, Ihab RK, Hailiang $L$, Jianjun $Q$, et al. Does GRASP affect DCE-MRI quantitative parameters and texture analysis in patients with esophageal cancer receiving preoperative neoadjuvant chemotherapy? Chinese J Acad Radiol. 2019;1(1):25-33.

18. Kuwano H, Nishimura Y, Oyama T, Kato H, Kitagawa Y, Kusano M, Shimada H, Takiuchi H, Toh Y, Doki Y, et al. Guidelines for diagnosis and treatment of carcinoma of the esophagus April 2012 edited by the Japan esophageal society. Esophagus. 2015;12:1-30.

19. Rice TW, Ishwaran H, Ferguson MK, Blackstone EH, Goldstraw P. Cancer of the esophagus and Esophagogastric junction: an eighth edition staging primer. J Thorac Oncol. 2017;12(1):36-42.

20. Mandard AM, Dalibard F, Mandard JC, Marnay J, Henry-Amar M, Petiot JF, Roussel A, Jacob JH, Segol P, Samama G, et al. Pathologic assessment of tumor regression after preoperative chemoradiotherapy of esophageal carcinoma. Cancer. 1994;73(11):2680-6.

21. Mukaka MM. Statistics corner: a guide to appropriate use of correlation coefficient in medical research. Malawi Med J. 2012;24(3):69-71.

22. Dijkhoff RAP, Beets-Tan RGH, Lambregts DMJ, Beets GL, Maas M. Value of DCE-MRI for staging and response evaluation in rectal cancer: a systematic review. Eur J Radiol. 2017;95:155-68.

23. Benz MR, Bongartz G, Froehlich JM, Winkel D, Boll DT, Heye T. Acceleration techniques and their impact on arterial input function sampling: nonaccelerated versus view-sharing and compressed sensing sequences. Eur J Radiol. 2018;104:8-13.

24. Wu AJ, Goodman KA. Positron emission tomography imaging for Gastroesophageal junction tumors. Semin Radiat Oncol. 2013;23(1):10-5.

25. Ilson DH, Minsky BD, Ku GY, Rusch V, Rizk N, Shah M, Kelsen DP, Capanu M, Tang L, Campbell J, et al. Phase 2 trial of induction and concurrent chemoradiotherapy with weekly irinotecan and cisplatin followed by surgery for esophageal cancer. Cancer. 2012;118(11):2820-7.

26. Chhabra A, Ong LT, Kuk D, Ku G, Ilson D, Janjigian YY, Wu A, Schöder H, Goodman KA. Prognostic significance of PET assessment of metabolic response to therapy in oesophageal squamous cell carcinoma. $\mathrm{Br} J$ Cancer. 2015;113(12):1658-65.

27. van Rossum PS, Fried DV, Zhang L, Hofstetter WL, Ho L, Meijer GJ, Carter BW, Court LE, Lin SH. The value of (18) F-FDG PET before and after induction chemotherapy for the early prediction of a poor pathologic response to subsequent preoperative chemoradiotherapy in oesophageal adenocarcinoma. Eur J Nucl Med Mol Imaging. 2017;44(1):71-80.

28. van Rossum PSN, van Lier ALHMW, van Vulpen M, Reerink O, Lagendijk JJW, Lin SH, van Hillegersberg R, Ruurda JP, Meijer GJ, Lips IM. Diffusionweighted magnetic resonance imaging for the prediction of pathologic response to neoadjuvant chemoradiotherapy in esophageal cancer. Radiother Oncol. 2015;115(2):163-70.

29. Elliott JA, O'Farrell NJ, King S, Halpenny D, Malik V, Muldoon C, Johnston C, Reynolds JV. Value of CT-PET after neoadjuvant chemoradiation in the prediction of histological tumour regression, nodal status and survival in oesophageal adenocarcinoma. Br J Surg. 2014;101(13):1702-11.

30. Heneghan HM, Donohoe C, Elliot J, Ahmed Z, Malik V, Ravi N, Reynolds JV Can CT-PET and endoscopic assessment post-Neoadjuvant
Chemoradiotherapy predict residual disease in esophageal Cancer? Ann Surg. 2016;264(5):831-8.

31. Li X, Conlin CC, Decker ST, Hu N, Mueller M, Khor L, Hanrahan C, Layec G, Lee VS, Zhang JL. Sampling arterial input function (AIF) from peripheral arteries: comparison of a temporospatial-feature based method against conventional manual method. Magn Reson Imaging. 2019;57:118-23.

\section{Publisher's Note}

Springer Nature remains neutral with regard to jurisdictional claims in published maps and institutional affiliations.
Ready to submit your research? Choose BMC and benefit from:

- fast, convenient online submission

- thorough peer review by experienced researchers in your field

- rapid publication on acceptance

- support for research data, including large and complex data types

- gold Open Access which fosters wider collaboration and increased citations

- maximum visibility for your research: over $100 \mathrm{M}$ website views per year

At BMC, research is always in progress.

Learn more biomedcentral.com/submissions 